International Aeronautical Congress, Earth Observation Symposium, Bremen, 2003

\title{
AN INTERFEROMETRIC SAR SATELLITE MISSION
}

\author{
Runge H.*, Gill E. **, Eineder M.*, S. Suchandt* \\ *Deutsches Zentrum für Luft- und Raumfahrt (DLR) e.V., Remote Sensing Technology Institute, \\ 82234 Wessling, Germany, hartmut.runge@dlr.de \\ **DLR, German Space Operations Center, 82234 Wessling, Germany, eberhard.gill@dlr.de
}

\begin{abstract}
The paper provides a critical review of the achievements in SAR interferometry from the ERS mission as well as from the Shuttle Radar Topography Mission SRTM. It describes the development from the original idea of the Interferometric Cartwheel to the concept of a formation flight of identical and active SAR satellites. From the experience gained from ERS and SRTM interferometric data processing as well as from the analysis of the Cartwheel concept a list of mission requirements has been set up. The most demanding one is the autonomous configuration flight of a tight $x$-band constellation, where the satellites fly as close as up to $30 \mathrm{~m}$ with a dead-band of $+/-10 \mathrm{~m}$. The guidance, navigation and control considerations come to the conclusion that such a mission is feasible.
\end{abstract}

\section{INTRODUCTION}

During the last decade the ERS satellites have pioneered Synthetic Aperture Radar (SAR) interferometry. Many new techniques and applications have been developed and numerous papers have been published. ${ }^{1}$ The great success of the ERS interferometry mission stems from its long term availability and stability of the instruments, the wise mission planning and operations at ESA, the stable attitude (at least for most of the lifetime of the satellites), relatively precise repeat orbits and highly accurate orbit data. Examples of the achievements are the images of seismic deformation patterns and glacier movement measurements.

The ERS-Tandem phase of ERS-1 and ERS-2 was the first SAR interferometry formation flight. The temporal decorrelation was reduced by a $24 \mathrm{~h}$ revisit time and the generation of digital elevation models became possible also over vegetated ground. Over forests, however, many data sets had to be averaged to gain a Digital Elevation Model (DEM) of acceptable quality.

The breakthrough for the DEM application was the Shuttle Radar Topography Mission (SRTM) in 2000 where single-pass interferometry with almost no decorrelation became possible. Highquality DEM mosaics of entire continents have been generated with the data from the JPL C- band radar and the DLR / ASI X-band radar which worked in parallel. ${ }^{2}$

Unfortunately the ERS era is comming to its end and the space shuttle Endeavour carrying the SRTM payload had to return to Earth after a 11 days spaceflight. The upcoming SAR satellites like ALOS, Radarsat- 2 and TerraSAR-X so far are single satellite missions. These systems will only allow for long time-lag repeat pass interferometry or for a very short time lag along track interferometry in split antenna mode using dual receivers.

However, the full potential of SAR interferometry will not be exploited by these missions although there is a clear demand for an operational long term dedicated Interferometric SAR (InSAR) mission.

Due to the limited physical dimensions of the spacecraft bus longer baselines can not be accomodated on a single satellite. Very lightweight deployable booms with a small secondary antenna on the tip may be a solution but the compensation of gravity gradient forces will require an advanced attitude control system. An alternative approach proposed in this paper is the formation flight of SAR satellites. In this distributed sensor concept two or more SAR sensors are located on different platforms. As a consequence the desired baselines can be adjusted in a flexible manner in the course of the mission depending on the user needs.

In order to form an across-track baseline the satellites must fly in parallel or with different altitudes. This is, however, impossible from an orbital mechanics point of view over long arcs of the orbit. Already in 1998 Massonnet came up with the solution. ${ }^{3}$ An "Interferometric Cartwheel" would enable to maintain a stable satellite configuration with appropriate acrosstrack baselines for the overall orbit. This idea has been further developed and detailed performance analysis are available. ${ }^{4}$ It has been pointed out that such reconfigurable clusters of SAR satellites will have also benefits for non-interferometric applications like resolution enhancement by the so called „superresolution technique" and wide swath. "Beside these European activities the TechSAT21 programme has been started in the US. ${ }^{6}$ 
As a further refinement for the SAR configuration flight this paper describes the benefits and techniques of an autonomous controlled configuration flight which is depicted in Fig. 1.

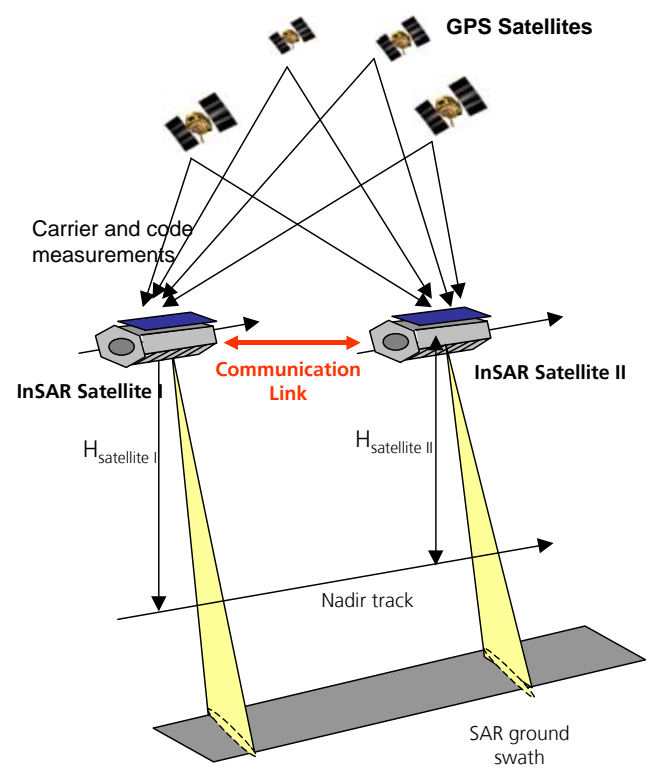

Fig.1. SAR satellite formation flight with GPS guided autonomous navigation system

The original proposal of the Interferometric Cartwheel included the idea of a "wheel" of passive micro-satellites flying in front or behind an existing SAR satellite acting as an „illuminator". However, we consider a self contained cartwheel of uniform but specially equiped active SAR minisatellites as easier to realize.

\section{LESSONS LEARNT FROM ERS AND SRTM}

In this chapter we will review the experience with ERS and SRTM interferometric data processing and we will summarize the requirements for $\mathbf{a}$ new interferometry mission.

a) For the application DEM generation the weakest point of the ERS-Tandem was the revisit time of one day. This long time span causes considerable decorrelation over forests and some agriculture fields. In order to minimize decorrelation effects the two interferometric data channels have to be acquired simultaneously in a single-pass and bi-static configuration.

b) A further disadvantage became evident when we tried to produce DEMs from steep mountains with ERS data. There it turned out that the foreshortening effect of the side-looking SAR geometry does not allow to produce high quality DEMs with the fixed and steep incidence angle of 23 deg of ERS. On the other hand the SNR is much lower at steep angles and increases rapidly with more shallow angles. If the incidence angle becomes too shallow in mountainous terrain shadowing appears. Eineder showed that the best compromise for alpine DEM generation is an incidence angle of 45 deg. ${ }^{7}$ However, a new system should have flexible incidence angles in order to be able to profit from the better SNR at steep incidence angles which can be used over moderate terrain.

c) ERS as well as SRTM had a fixed spatial resolution which lead to multi-look SAR images and DEMs with a sampling of $30 \mathrm{~m} \times 30 \mathrm{~m}$. The DEM quality was according to the DTED-2 specification. ${ }^{4}$ For some regions of the world DEMs with a better sampling, e. g. $10 \mathrm{~m} \times 10 \mathrm{~m}$ (DTED-3), however, are required. Modern SAR satellites like TerraSAR-X and Radarsat-2 offer high resolution modes which can provide the desired quality. ${ }^{8}$ Therefore, the SAR for the new InSAR configuration shall have a single look spatial resolution of approximately $3 \mathrm{~m}$ in order to perform multi-looking for noise suppression.

d) SRTM has mapped the Earth land mass between 60 deg $\mathrm{N}$ and 57deg $\mathrm{S}$ in just 11 days. This has been possible due to the extremely powerfull Shuttle fuel cells which delivered more than $900 \mathrm{kWh}$ of electical energy as well as very robust radar systems which have been able to be in operation for data takes longer than 30 min in each Earth orbit. The instrument data rate was $180 \mathrm{Mbit} / \mathrm{s}$ for the C-band radar and 90 Mbit/s for the X-band radar which can't be downlinked to a single ground station with current technology. Therefore, SRTM had to rely on on-board tape recorders. This situation will aggravate with a high resolution SAR system which requires more energy and therefore normally has a reduced swath width. Due to these technical bottlenecks a future satellite system should have a lifetime of several years in order to be able to relax the requirements on the instrument and the data downlink. With a satellite mission large parts of the world can be covered in high resolution and mission phases dedicated to different applications can be performed.

Furthermore, with the availability of high resolution DEMs it becomes interesting to repeat the measurements after a longer time span in order to detect 3D-changes. In climate change studies for example it would be of interest to perform regularly a three-dimensional stock taking of representative glaciers worldwide. It is therefore highly desirable that the satellites of the proposed misson have an expected mission lifetime in the order of 5 to 8 years. 
Furthermore, they shall be placed into a polar orbit in order to cover the polar regions.

e) SAR interferometry exploits the differential phase between two observations. Hence the stability of the reference wave field generated by the local ultra stable oscillator is a critical parameter. In the SRTM configuration only one oscillator was used, but in a formation flight of SAR sensors we have to deal with more oscillators. A drift between the oscillators would cause errors in the interferogram. Especially for bistatic SAR interferometry and for long data takes a synchronisation between the oscillators is mandatory. ${ }^{9}$ Fortunately it exists an elegant method to synchronize the receivers. ${ }^{10}$ Ground control points can be avoided and standard oscillators can be used if the oscillator signals are exchanged between the SAR instruments by an inter-satellite link.

f) Errors in the measurement of the baseline length and the orientation of the baseline in space directly cause errors in the digital elevation model. It has been shown that for an $X$-band system the orbits of the satellites in the formation must be known with an accuracy of 1 $\mathbf{m m}$ or better.

g) The major findings of the operational X-SAR SRTM data processing were concerned with mountainous terrain, were the final DEM data quality was partly deteriorated at steep mountain slopes. This was caused by problems with the phase-unwrapping (see next paragraph) and with the weak signal from mountain slopes not oriented towards the sensor.

The SAR geometry is necessarily side looking and in order to avoid foreshortening effects the incidence angle must be in the order of $45 \mathrm{deg}$ or even shallower. As a result, the data from the slopes not oriented towards the sensor appear noisy and decorrelated. In order to overcome this problem, mountains have to be observed by different look directions. „The look around the mountains" can be accomplished by the use of ascending and descending orbits but for mountainous terrain a left- and right looking capability of the instrument is mandatory.

h) The parameters of fundamental importance for SAR interferometry are the baseline orientation (along- or across track) and the baseline length. Depending on the application and the terrain in focus the baseline needs to be adjusted properly.

With ERS and SRTM this has been possible only to a certain extend. Most ERS orbits have been kept within a baseline range of $300 \mathrm{~m}$ but within these limits they appear to be randomly distributed Many scientific results of ERS interferometry have only be achieved because of the availability of the immense stock of ERS data, special search tools made available by ESA and the flexibility of the scientists in terms of test site selection. ${ }^{11}$ In a new InSAR mission it is highly desirable that the different InSAR techniques can be used operationally. The ultimate goal for many users would be the ability to request in addition to the scene a certain baseline. However, in a practical mission the user interests have to be grouped together and the mission has to be organised in phases. Nevertheless, the requirement remains that it must be possible to adjust a certain baseline. Beside DEM generation, highly demanding applications are SAR tomography and superresolution techniques where for each acquisition the baseline has to be incremented. 5,12

The mast of SRTM has been $60 \mathrm{~m}$ long and it has been kept always in the same attitude. At the phase unwrapping stage of the X-SAR data processing, it turned out that it was sometimes impossible to unwrap the phase properly over scenes of highly mountainous terrain. A baseline of half the length would have been required over steep mountains. On the other hand a much better vertical resolution could have been obtained with longer baselines.

From the experience gained for X-SAR SRTM data processing it has been found that for a $X$ band $(9.6 \mathrm{GHz}, \quad 0.031 \mathrm{~m}$ wavelength) SAR satellite formation working in the bi-static mode in an orbit altitude of $600 \mathrm{~km}$ at least two different baselines have to be adjusted in order to cover the different terrain types. ${ }^{9}$ A short baseline for strong topography with a height of ambiguity of $500 \mathrm{~m}$ and a longer baseline for moderate topography with a height of ambiguity of $50 \mathrm{~m}$ have to be foreseen. The corresponding desired baselines are approximately $40 \mathrm{~m}$ and $400 \mathrm{~m}$, respectively. If we request an accuracy of only $+/-20 \%$ the baselines must be adjusted with $+/-8 \mathrm{~m}$ accuracy for strong topography and $+/-80 \mathrm{~m}$ for moderate topography.

An L-band (1.3 GHz, $0.24 \mathrm{~m}$ wavelength) configuration for the same orbit altitude and the same heights of ambiguity we need a baseline of app. $300 \mathrm{~m}$ (with a +/- $60 \mathrm{~m}$ dead-band) and for the moderate topography a baseline of app. $3,000 \mathrm{~m}$ ( with a dead-band of $+/-600 \mathrm{~m}$ ).

For the application of differential interferometry across track baselines should be avoided because the ground topography would introduce phase errors into the measurement of movements or ground subsidences. ${ }^{13}$ The phase errors can be compensated if a precise reference DEM is available. This is not always the case and therefore it is required that for mission phases with differential interferometry an orbit dead-band of $+/-10 \mathrm{~m}$ in the X-band 
and about $+/-80 \mathrm{~m}$ in the L-band are maintained.

For the monitoring of ocean currents, small time lags in the order of milli-seconds between the two oberservations are required. The time lag must not exceed certain limits for $X$ - and L-band because the ocean surfaces decorrelates. Therefore, also in this case a tight configuration of the satellites is required. In X-band the spacecrafts shall be separated by $30 \mathrm{~m}$ with a $+/-$ $10 \mathrm{~m}$ deadband and separated $225 \mathrm{~m}$ with $+/-75$ $\mathrm{m}$ dead-band in L-band. 9,14

In summary, the orbit control for an X-band configuration must cope with the situation that two spacecraft fly at a nominal distanc of $30 \mathrm{~m}$ and orbit deadbands of $+/-10 \mathrm{~m}$ must be kept. An L-band configuration will have a nominal distance of $300 m$ with $\mathrm{a}+/-80 \mathrm{~m}$ dead-band.

\section{GUIDANCE, NAVIGATION AND CONTROL CONSIDERATIONS FOR INSAR MISSIONS}

Having proposed a mission scenario from the users point of view, the following analysis focuses on how such demanding requirements can be met.

\section{Conceptual and Guidance Aspects}

Common to all scenarios discussed above is the potential to operate the space segment in a largely autonomous manner. To that end, the implementation of capable navigation sensors together with adequate thrusters and an advanced autonomous onboard navigation system enables the efficient operations of the space segment with a minimum support from the ground control center. Relying on an intersatellite communication terminal for the exchange of navigation data, the satellites would be able to autonomously control their relative orbits by applying appropriate orbit maneuvers. Moreover, both tight and loose SAR formations enable missions which are not constrained to a particular baseline. Instead, the baseline may, within certain limits, be adjusted freely or changed in a flexible and dynamic way during the mission lifetime.

\section{Flight Dynamics and Navigation Sensors}

Neither close nor loose formation flight poses, from a pure flight dynamics point of view, a basic problem. However, any two bodies separated in space will experience differential accelerations, which may give rise to a complex relative motion. The problem of relative motion between two spacecraft may be treated in an analytic manner using the Clohessy-Wiltshire or Hill's equations for circular orbits in the two-body approximation with separation distances of less than $1 \mathrm{~km} .^{15}$ For more realistic scenarios, the numerical integration of the equations of motion for the two satellites and their subsequent differencing yields a precise approach to relative position and velocity computation. It is obvious that spacecraft with similar shapes and masses and small separations experience less differential accelerations than spacecraft with different shapes and masses and large separations due to perturbation forces which act in addition to the Newtonian gravity.

Regarding the orbit reconstruction accuracy, the requirements from SAR processing for the a posteriori reconstruction must be distinguished from the real-time orbit determination requirements for autonomous on-board orbit control. For tight $\mathrm{ATI}$, the required a posteriori accuracy may be derived from present-day phase noise figures and the SAR wavelength, which yields about $1 \mathrm{~mm}$ in X-Band and $7 \mathrm{~mm}$ in L-Band. ${ }^{17}$ Concerning the control of a close formation, we assume as an order of magnitude estimation that for safe operations of a formation separated by a distance $x$ a control accuracy of $x / 10$ and a sensor accuracy for relative motion of $x / 100$ is required. For close formations, this estimate is equivalent to $0.3 \mathrm{~m}$ in X-Band and $2 \mathrm{~m}$ in L-Band.

Navigation sensors for formation control thus have to provide an accuracy of at least $0.3 \mathrm{~m}$. Recent simulations of real-time orbit determination for formations with an $800 \mathrm{~m}$ baseline have revealed relative position errors as low as $1 \mathrm{~mm}$ using common single frequency GPS receivers. ${ }^{18}$ Although the relative position error increases with the formation baseline, it is expected that the navigation and control requirements for loose formations still can be met using GPS receivers as long as the frequent exchange of mutual navigation data between the satellites is guaranteed.

\section{Control Approaches and Actuators}

The satellite orbit control is essentially based on the knowledge of the absolute position and velocity information of each satellite and of its the co-orbiting partner. To that end, the formation has to be equipped with a communication terminal which allows the mutual exchange of GPS-based navigation data collected at each satellite. The onboard orbit determination function of the autonomous navigation system allows in turn to derive precise and robust absolute and relative position and velocity information for each satellite, based on a suitable data combination scheme, data screening and smoothing as well as a bridging of data gaps. 
Based upon the spacecraft absolute and relative position and velocity as well as a suitable guidance law, the activation of the spacecraft actuators, i.e. thrusters, for orbit control may be computed and executed autonomously onboard.

To that end, the autonomous onboard navigation system will perform a transit to a specific userdefined baseline only from stable formation flying geometry, such as those defined by Cartwheel or Pendulum. This approach does not only provide the necessary robustness for safe spacecraft operations but will also minimize fuel consumption, since only those formation geometries are realized, which do not require maneuvers in the absence of differential accelerations.

The autonomous absolute and relative orbit control implies not just a reduction of the ground operations manpower but, for specific mission scenarios with frequent maneuvers or tight separation, it is an indispensable requirement.

Generic autonomous formation keeping requires besides a powerful onboard processor an attitude control system which delivers absolute (and possibly relative) attitude information which is not deteriorated in any spatial direction. Furthermore, to obtain the full flexibility for general orbit maneuvers without the need for time-consuming and complicated spacecraft rotations, the orbit actuators should be able to provide thrust in all six spatial directions.

Considering the case of a close formation with a +/- 10m orbit dead-band, typical formation keeping maneuvers would require velocity increments of about $5 \mathrm{~mm} / \mathrm{s}$ and a velocity resolution of $1 / 20(5 \%)$ or $0.3 \mathrm{~mm} / \mathrm{s} .{ }^{17}$ Assuming furthermore a total spacecraft mass of $1200 \mathrm{~kg}$, as for the TerraSAR-X satellite, this translates into an impulse transfer per maneuver of $4.2 \mathrm{Ns}$ with a resolution of $0.2 \mathrm{Ns}$, which could be achieved e.g. with a $5 \mathrm{~N}$ thruster firing for $1 \mathrm{~s}$ or a $14 \mathrm{mN}$ thruster firing for $300 \mathrm{~s}$. Whether a quasi-continuous thrusting, as provided by an electrodynamic thruster system, is suitable for the envisaged applications has not yet been addressed in the literature. For a tight ATI formation flying, thruster systems with thrust levels of $10-500 \mathrm{mN}$ appear as most appropriate. A preliminary fuel budget estimate indicates a fuel consumption of about $60 \mathrm{~kg}$ for the formation acquisition and a 2 years formation keeping in the $+/-10 \mathrm{~m}$ dead-band. However, the larger part of the mission will be performed in the $+/-70 \mathrm{~m}$ dead-band where the fuel consumption is very much relaxed.

\section{THE NEW INSAR MISSION}

Due to the great importance and success of SAR interferometry a new mission is proposed which will make use of a formation of SAR satellites specially designed for this purpose. The mission will consist of at least two to three identical SAR satellites with left/right looking and multi-incidence angle capability. The radar systems can be synchronized in order to support single-pass and bi-static operational modes. The exact orbit track will be determined by GPS measurements and on-board post-processing. The orbit data will be interchanged within the configuration by an inter-satellite data link. An autonomous guidance system will take care of appropriate baselines and a stable orbit configuration.

At least two satellites will fly in a close formation to perform across-track interferometry for the generation of digital elevation models or for along-track interferometry to image ocean currents or traffic flows. For differential interferometry applications like the measurement of glacier flow fields or grounding line detection in the arctic shelf ice, a third satellite will follow a partner satellite in exactly the same orbital track in order to avoid undesired large across-track baselines. The time-lag between the two observations will be adjustable.

Differential interferometry applications, like ground subsidence measurements and the detection of displacement patterns from earthquakes, require long time lags between the observations (multiples of the complete orbit cycle). In this case no exchange of GPS information between the satellites is necessary because the satellites will automatically follow reference orbits stored in the computer memory of the navigation system.

\section{CONCLUSION}

A novel concept for an interferometric SAR mission is proposed which overcomes shortcomings of former missions. It consists of a formation of identical SAR satellites which shall be guided by an autonomous orbit control system. This concept allows to precisely adjust the desired interferometric baselines in a flexible and dynamic way depending on the users needs and during the course of the mission. In addition these measures will improve the final product quality of many interferometric applications like the generation of digital elevation models. Furthermore, for certain interesting techniques like SAR tomography, super-resolution imaging and the imaging of ocean currents this approach paves the way from technology demonstration to operational use. 
International Aeronautical Congress, Earth Observation Symposium, Bremen, 2003

However, a flexible baseline adjustment combined with efficient spacecraft operations requires a careful design of the space segment. This comprises an adequate GPS receiver, an intersatellite communication terminal for the exchange of GPS navigation data, a powerful attitude and orbit control system and adequate thrusters which allow to deliver thrust in all six directions.

First studies on a tight ATI mission and real-time hardware-in-the-loop simulations of autonomous formation control have revealed the feasibility of the proposed approach. ${ }^{17,18}$

\section{ACKNOWLEDGMENT}

The authors like to thank the CNES CartWheel team headed by F. Douchin, the DLR CartWheel team headed by $G$. Krieger and F. Jochim as well as R. Romeiser for the valuable discussions.

This work has been supported by the European Space Agency, ESA-ESTEC, Noordwijk, The Netherlands, under contract 16100/02/NL/EC.

\section{REFERENCES}

1. http://earth.esa.int/workshops/fringe03/

2. Web pages of the Shuttle Radar Topography Mission SRTM:

http://www.jpl.nasa.gov/srtm/, http://www.dfd.dlr.de/srtm/ http://srtm.det.unifi.it/index.htm

3. Massonnet D., Capabilities and limitations of the interferometric cartwheel, IEEE TGRS, 2001, 39, (3), pp. 506-520

4. Krieger G., Fiedler H., Mittermayer J., Papathanassiou K., Moreira A., Analysis of multistatic configurations for spaceborne SAR interferometry, IEE Proc.-Radar Sonar Navig., Vol 150, No. 3, June 2003

5. Runge $H_{\text {., }}$ Werner M., Eineder M., Breit $H_{\text {., }}$ Suchandt S., Massonnet D., Advanced Synthetic Aperture Radar Observations with Clusters of SAR Satellites, ISPRS Workshop "High Resolution Mapping From Space”, Sep. 19-21, 2001, Hannover, Germany

6. TechSat21homepage:http://www.vs.afrl.af.mil/Tec hProgs/TechSat21/

7. Eineder M., Holzner J., Interferometric DEMs in Alpine Terrain - Limits and Options for ERS and SRTM, Proceedings of IGARSS 2000, Honolulu, Hawaii, USA, July 2000

8. Rabus B., Eineder E., Roth A., Bamler R., The shuttle radar topography mission - a new class of digital elevation models acquired by spaceborne radar, ISPRS Journal of Photogrammetry \& Remote Sensing, 57, 2003, page $241-262$
9. New Techniques For Simultaneous SAR Interferometry, ESA ESTEC, Contract No: $16100 / 02 / \mathrm{NL} / \mathrm{EC}$, study currently under preparation

10. Eineder M., Oscillator Clock Drift Compensation in Bistatic Interferometric SAR, Proceedings of IGARSS 2003, Toulouse

11. Solaas G., Coulson S. N., ERS-1 SAR Interferometry Orbit Listing User Note, ESA I ESRIN, online available: http://earth.esa.int/rootcollection/eeo4.10075/0010 $\underline{\text { d.html }}$

12. Reigber A., Moreira A., First Demonstration of Airborne SAR Tomography Using Multibaseline LBand Data, IEEE Transactions onGeoscience and Remote Sensing, Vol. 38, No. 5, IGRSD2, 2000, page $2142-2152$

13. Alessandro Ferretti, Claudio Prati, and Fabio Rocca. Nonlinear subsidence rate estimation using permanent scatterers in differential SAR interferometry. IEEE Transactions on Geoscience and Remote Sensing, 38(5):2202-2212, September 2000.

14. Romeiser R., Breit H., Eineder M., Runge $H_{\text {., }}$ Flament P., de Jong K., Vogelzang J.; Validation of SRTM-Derived Surface Currents off the Dutch Coast by Numerical Circulation Model Results; Proceedings of IGARSS, July 21-25 2003, Toulouse (2003).

15. Clohessy W.H., Wiltshire R.S.; Terminal Guidance System for Satellite Rendezvous; Journal of the Aerospace Sciences, 27; 653-658 (1960).

16. Leung S., Montenbruck O.; High Precision RealTime Navigation for Spacecraft Formation Flying; ION GPS 2003 Conference, 9-12 Sept. 2003, Portland, Oregon (2003).

17. Gill E., Runge H.; Tight Formation Flying for an Along-track SAR Interferometer; IAC-03-A.3.03; 54th International Astronautical Congress (IAF), Sep. 29 - Oct. 3, 2003, Bremen, Germany (2003).

18. Gill E., Naasz B., Ebinuma T.; First Results from a Hardware-in-the-Loop Demonstration of ClosedLoop Autonomous Formation Flying; 26th Annual AAS Guidance and Control Conference, February 5-9, 2003, Breckenridge, CO (2003). 